\title{
Droits à la retraite et mortalité différentielle
}

\author{
Antoine Bommier* Thierry Magnac ${ }^{\dagger} \quad$ Benoit Rapoport ${ }^{\ddagger}$ \\ Muriel Roger ${ }^{\S}$
}

Première version: Avril 2003.

Cette version: Mai 2004

\section{Résumé ${ }^{1}$ :}

Nous utilisons des données administratives, l'Echantillon Interrégimes des Retraités, pour estimer la mortalité différentielle selon le montant des pensions de retraite en France. Ces données concernent certaines générations nées entre 1906 et 1930 et leur mortalité est évaluée entre 1997 et 2001. Nous utilisons diverses méthodes d'estimation et distinguons plusieurs sous-échantillons suivant le sexe ou d'autres caractéristiques individuelles pour dériver nos résultats les plus robustes. La mortalité différentielle est significative pour les hommes mais ne l'est pas pour les femmes. Dans la population masculine que nous considérons, un doublement du revenu entraine une augmentation du taux instantané de mortalité d'environ $10 \%$. Nous complétons cette mesure par l'évaluation illustrative suivante. La mortalité différentielle ne semble pas remettre en cause le caractère redistributif, d'un point de vue monétaire, du système de pensions de retraites en France mais elle semble affecter significativement la magnitude de la redistribution.

*GREMAQ-Université de Toulouse et INRA Paris Jourdan. Antoine.Bommier@univ-tlse1.fr. http://www.inra.fr/ESR/UR/lea/bommier.htm

†INRA, CREST-INSEE et IDEI. Aussi affilié avec le CEPR. ENS Thierry.Magnac@ens.fr. Web: http://www.inra.fr/ESR/UR/lea/magnac.htm

$¥$ DREES, Ministère des Affaires Sociales, TEAM, Université de Paris 1, et INRA Paris-Jourdan, BENOIT.RAPOPORT@SANTE.GOUV.FR

§INRA Paris-Jourdan Muriel.Roger@ens.fr.Web: http://www.inra.fr/ESR/UR/lea/roger.htm

${ }^{1}$ Nous remercions Emmanuelle Cambois de nous avoir fait bénéficier de sa connaissance extensive des études démographiques sur la mortalité différentielle, deux rapporteurs anonymes et les participants aux Journées 2003 de l'AFSE pour leurs commentaires. Nous remercions la DREES pour le financement 01-140011 et l'accès aux données EIR. Aucun de nos interlocuteurs ne peut être tenu pour responsable des erreurs et omissions que nous aurions commises. 


\section{Introduction}

Il est bien connu que les risques de décès varient sensiblement selon les conditions socioéconomiques des individus. En France, toutefois, l'essentiel des études sur les inégalités sociales face à la mort se sont concentrées sur les différences de mortalité selon les professions ou catégories socio-professionnelles (CSP). On trouve une longue et riche tradition de tels travaux, avec notamment ceux de Bertillon (1889), Huber (1912), Febvay et Aubenque (1957) Lederman (1960), Desplanques (1973, 1991 et 1993) et Mesrine (1999). Mais, alors que les différences de mortalité selon le revenu ont été documentées dans différents pays, comme les Etats-Unis, le Canada ou le Royaume-Uni ${ }^{2}$, on ne trouvait pas encore d'études de l'influence du revenu ou de la richesse sur la mortalité en France. Cette information est pourtant des plus intéressantes, tant pour comprendre les déterminants de la mortalité, que pour être à même de calibrer une politique redistributive dans un contexte de mortalité hétérogène.

Cet article, comme les travaux de Jusot (2003), vise à combler cette lacune. On peut principalement utiliser deux corpus de données pour estimer la mortalité différentielle; utiliser, d'une part, des données longitudinales qui suivent certains individus au cours du temps et indiquent s'ils étaient en vie ou décédés à différentes dates; d'autre part comparer la distribution des personnes décédées à la distribution de la population des vivants. Chacune des méthodes a ses avantages et ses inconvénients. L'approche longitudinale est séduisante, mais les taux de décès étant relativement faibles, elle est fragile puisqu'elle doit s'appuyer sur des données longitudinales ne souffrant pas (ou très peu) d'attrition. En particulier il devient peu raisonnable d'utiliser des enquêtes ménages en panel où les taux d'attrition liés à la mobilité ou d'autres causes sont élevés et difficiles à distinguer de l'attrition par décès. Il faut donc s'appuyer sur les rares données où l'attrition n'empêche pas l'estimation des taux de décès. C'est ce que nous faisons dans cet article en utilisant l'Echantillon Interrégimes de Retraités (EIR) où il n'y a aucune attrition pour des raisons autres que le décès puisque la présence, ou l'absence, de la base est donnée par le versement ou non, de la pension de retraite. Ces données sont particulièrement précises mais ont pour double inconvénient de ne pas être représentatives de la population de la France ${ }^{3}$ et de ne contenir que peu d'informations utilisables pour une analyse multivariée. La seconde méthode est celle utilisée par Jusot (2003) qui compare les données de l'enquête Patrimoine au Décès

\footnotetext{
${ }^{2}$ On pourra se reporter aux travaux récents de Adams et al. (2003) pour les Etats-Unis, de Mustard et al (1997) pour le Canada et de Attanasio et Emmerson (2003) pour le Royaume-Uni. Une bibliographie complète des travaux sur des données des Etats-Unis, est présentée par Adams et al. (2003). Toutes ces études indiquent qu'il y a une corrélation négative entre mortalité et revenu.

${ }^{3}$ L'EIR est un échantillon de pensionnés des principaux régimes de retraite. Il ne permet donc pas de mesurer la mortalité différentielle dans la population des personnes qui n'ont pas ou peu travaillé ou des individus affiliés à certains régimes de retraite, il est vrai, résiduels.
} 
(représentatif des décédés) aux données des Revenus Fiscaux (représentatifs des vivants). Ces enquêtes contenant plus d'informations que celle que nous utilisons, Jusot peut aborder un plus grand nombre de questions. Toutefois, cette approche est limitée, comme cela est expliqué dans Jusot (2003), par le fait que les données utilisées ne sont qu'imparfaitement comparables ${ }^{4}$.

En utilisant les données de l'EIR nous pouvons estimer la relation entre taux de mortalité des retraités et montant de leur pension. Cette estimation est particulièrement robuste puisque les données issues de registres administratifs ne souffrent d'aucun des problèmes habituels d'attrition, d'erreurs de mesure ou de non réponses sur le montant des pensions. Nous trouvons que la mortalité différentielle selon le niveau de pension est significative pour les hommes. Nous obtenons une élasticité des taux de mortalité au niveau des pensions qui varie selon l'âge. Elle est l'ordre de $-0,5$ à 70 ans et de $-0,3$ à 80 ans. Cela impliquerait une élasticité revenu de l'espérance de vie à 60 ans de l'ordre de 0.18 , ce qui est loin d'être négligeable. En revanche, les résultats pour les femmes ne sont significatifs qu'à certains âges et sous certaines hypothèses. Cela résulte sans doute de la structure de notre échantillon qui en n'incluant que des individus affiliés à une caisse de retraite, opère une sélection importante pour les femmes.

Cette base de données contient néanmoins peu de renseignements sur d'autres caractéristiques individuelles que le revenu et peu de renseignements sur les caractéristiques familiales, à part le nombre d'enfants et la présence ou non de pensions de reversion. Il nous est de fait difficile de dissocier les différents effets qui peuvent aboutir à la relation entre mortalité et pensions que nous observons. En particulier, nous n'avons aucune information sur l'état de santé initial des individus. Or, les causalités croisées entre statut socio-économique, comme le montant des pensions, et santé, dont la mortalité, ont fait l'objet d'âpres débats parmi les épidémiologistes suite à deux célèbres études de Whitehall sur les fonctionnaires britanniques (Marmot et al., 1991). Ces débats sont repris, depuis quelques années, par des économistes qui utilisent des méthodes empiriques originales, et ceci afin de mieux comprendre les comportements d'épargne et de consommation aux âges élevés. Les arguments de ces débats sont remarquablement bien résumés par Smith (1999). Il y est expliqué qu'interpréter nos résultats comme la mesure d'une causalité du revenu, ou de la richesse, vers la mortalité néglige diverses sources de causalité rétroactive. Des accidents de santé, ou la révélation d'une maladie chronique, durant la vie active peuvent faire que les revenus sont plus faibles et les carrières interrompues. Des frais médicaux importants peuvent grever le patrimoine.

\footnotetext{
${ }^{4}$ Une des difficultés rencontrées par Jusot est par exemple que l'échantillon de l'enquête Patrimoine au décès est représentatif de la population des individus alors que ceux des Revenus Fiscaux sont représentatifs de la population des ménages fiscaux.
} 
Certains auteurs avancent enfin que la perspective de vivre moins longtemps accélére la décumulation du patrimoine aux âges élevés et diminue la probabilité de faire un legs à ses descendants.

Utiliser une mesure des droits à pension pour mesurer le statut socio-économique, comme nous le faisons, évite certains de ces écueils même s'ils restent une mesure un peu lointaine de la richesse puisqu'ils différent suivant les régimes, publics ou privés par exemple. Nous ne disposons pas non plus d'informations sur le revenu familial. Néanmoins, ces droits à pension sont moins sensibles à l'état de santé que le patrimoine global sauf dans la mesure où ils reflètent une capacité à générer des revenus plus faibles, tout au long de la carrière, ou des départs en retraite plus précoces. De plus, n'utiliser des données que sur des retraités permet de s'abstenir de distinguer les effets de revenu des effets directs du statut sur le marché du travail sur la mortalité (Snyder et Evans, 2002). La corrélation positive entre état de santé général et revenu ne cesse en effet de croître qu'au départ en retraite (Deaton \& Paxson, 1998). Utiliser les pensions de retraites des retraités pour mesurer leur statut socio-économique en contrôlant pour les durées de cotisation comme nous le faisons, n'écarte pourtant pas toutes les possibilités de causalité inverse. En admettant que l'état de santé général est déterminé dès l'enfance et que le revenu dépend de l'état de santé à l'âge adulte, le montant des pensions pourrait être déterminé par la même cause commune que la mortalité. Plus généralement d'ailleurs, il reste extrêmement difficile d'expliquer la mortalité différentielle par les arguments habituels, comme les conduites à risque (tabac et comportements alimentaires par exemple) ou comme l'accès aux soins de santé, qui certes sont anticorrélées au revenu mais de manière insuffisante pour expliquer l'impact du revenu (études de Whitehall, Marmot et al., 1991). Ceci semble valoir aussi pour la dangerosité des professions même si une enquête sur les fonctionnaires est moins à même de répondre à une telle question. Un nouvel élément du débat, apporté par Adams et al. (2003), montre qu'aux âges élévés - plus de 70 ans- les conditions socio-économiques ne "causent" pas (au sens de Granger) les chocs sur la santé, comme l'apparition de nouvelles maladies. ${ }^{5}$ Les résultats sont similaires en utilisant des données américaines, britanniques ou suédoises.

En conclusion, la relation entre revenu et mortalité est donc essentiellement une relation statique mal expliquée. Elle conditionne pourtant de nombreux diagnostics sur les politiques publiques et en particulier sur le système de pensions de retraite. C'est pourquoi, en prenant la mortalité différentielle comme une donnée, nous discutons aussi, dans ce papier, de son impact sur l'analyse des systèmes de retraites. Nous estimons la redistribution que peut engendrer cette mortalité différentielle ainsi que l'hétérogénéité des incitations qu'il faudrait

\footnotetext{
${ }^{5} \mathrm{~A}$ une exception près que sont les maladies mentales mais l'interprétation de cet effet reste mystérieuse.
} 
mettre en place pour que le mode de calcul des montants de pensions soit actuariellement neutre, en dépit de cette mortalité différentielle. Cette discussion qui s'appuie sur les valeurs estimées des élasticités permet de donner les ordres de grandeur des différents phénomènes liés à la mortalité différentielle.

Dans la section 2, nous présentons les données que nous utilisons et l'estimation nonparamétrique de l'influence de la richesse sur la mortalité. Nous reportons dans la section suivante des estimations paramétriques d'une telle relation en contrôlant pour d'autres variables explicatives et d'autres causes de mauvaise spécification. Nous évaluons dans cette section de manière générale la robustesse de nos mesures d'impact et nous montrons que l'effet des pensions va au-delà de l'effet des CSP, il est vrai mesuré de façon grossière. Nous illustrons dans la dernière section l'impact de nos mesures sur le caractère redistributif du système de pensions en France.

\section{Données et estimations non-paramétriques}

\subsection{Les données}

Les données utilisées sont extraites de deux vagues successives de l'Echantillon Interrégimes de Retraités en 1997 et 2001 (voir DREES, 1996). Construites à partir des déclarations exhaustives des caisses de retraite qui sont appariées à des données sur les salaires et les périodes de chômage, elles donnent des informations sur les caisses de retraite, le montant de la retraite et sa ventilation entre les différents types d'avantages, en droits directs ou en droits indirects, le nombre de trimestres d'assurance et un nombre réduit d'autres caractéristiques individuelles. Dans cet échantillon, il est très rare de liquider ses droits après 67 ans. Nous nous sommes donc restreints aux générations nées en 1930 et avant (l'enquête se fait pour les générations en 1906, 1912, 1918, 1922, 1926) pour qu'à quelques exceptions près, tous les individus nés les 6 premiers jours d'octobre de ces années, qui ont travaillé et cotisé à une caisse de retraite et qui sont en vie en 1997 soient présents dans notre base de données. Les exceptions concernent les retraités de France non-métropolitaine, qui ne sont pas présents en 1997 mais le sont en $2001^{6}$. A côté de cela, quelques retraités ayant liquidé avant 1997 ne sont enregistrés qu'en 2001 et non en 1997 car ils avaient été “oubliés” en 1997. Il n’en reste pas moins que si un individu est présent dans la base en 1997, la seule raison de son absence en 2001 est son décès. Nous obtenons ainsi une mesure, d'excellente qualité, de la probabilité de survie à quatre ans (entre 1997 et 2001) pour des générations d'âge compris entre 67 et 91 ans. Il n'y a en effet aucun des problèmes d'attrition que l'on rencontre dans les

\footnotetext{
${ }^{6}$ En toute rigueur ce sont les personnes nées en France non-métropolitaine. En revanche, les personnes nées en métropole et résidents à l'étranger sont présents.
} 
enquêtes ménages utilisées habituellement pour évaluer la mortalité différentielle (Attanasio et Emmerson, 2003).

D'autre part, il existe une multitude de caisses de retraite en France et les modes de gestion des pensions de ces caisses diffèrent assez fortement. C'est pourquoi, le champ que nous retenons inclut les salariés du secteur privé, Caisse Nationale Assurance Vieillesse (CNAV) et Mutuelle Sociale Agricole (MSA) pour les salariés agricoles, les salariés des régimes "spéciaux" $^{7}$ et les salariés de la fonction publique et des collectivités locales ayant au moins un avantage de base de retraite en droit direct. Nous définissons comme principale, la caisse qui, premièrement, verse une pension de droit direct, deuxièmement, verse la contribution la plus importante dans le montant total de la pension de retraite et troisièmement, a reçu du salarié des cotisations pendant 60 trimestres au moins. Nous nous sommes restreints aux individus qui, d'après notre définition, ont une caisse principale. La coupure à 60 trimestres est arbitraire à ceci près qu'elle correspond à la longueur de cotisation minimale pour recevoir une pension dans le secteur public (15 ans). En dessous de 60 trimestres, la vie active pourrait avoir été assez instable. De toute façon, nous contrôlons en général nos estimations par le nombre de trimestres de cotisations et nous avons fait varier cette sélection pour nous assurer de la robustesse de nos résultats. Pour les hommes, cette restriction aux individus ayant travaillé suffisamment au cours du cycle de vie ne semble pas poser de sérieux problèmes, alors que, pour les femmes, elle entraine une sélection importante qui, on le verra, se retrouve sans doute dans nos résultats.

La bonne qualité de la variable de richesse ou de statut socio-économique est également à souligner puisque nous utilisons surtout les droits directs à la retraite reportés par les caisses de retraite. Comme nous l'avons déjà mentionné, la relation que nous recherchons est une relation entre survie et revenu moyen de cycle de vie. Au contraire des revenus mesurés dans les enquêtes ménages, les droits à la retraite ne sont pas un revenu transitoire obtenu par déclaration vers la fin de la vie active, période qui est fréquemment troublée par des périodes de chômage, de préretraite ou de changements d'horaires de travail (Bommier, Magnac et Roger, 2003). Dans cet échantillon, on peut néanmoins construire aussi d'autres notions de revenu en utilisant par exemple le montant total de la retraite qui intègre les droits indirects à des pensions de retraite (enfants, reversion et circonstances spéciales) ou l'indice du traitement pour les fonctionnaires. Nous utiliserons de tels indicateurs pour évaluer la robustesse de nos résultats.

Pour finir cette description, il n'y a que peu d'autres variables individuelles, le sexe, la région de résidence, le nombre de trimestres de cotisation et la caisse de retraite et aucune

\footnotetext{
${ }^{7}$ dont les principaux sont EDF-GDF, la RATP, la SNCF et la Banque de France.
} 
variable familiale. Les informations professionnelles sont assez réduites puisqu'elles consistent en 5 catégories socio-professionnelles (cadres supérieurs, professions intermédiaires, employés, ouvriers qualifiés, ouvriers non qualifiés).

Le tableau 1 reporte les moyennes des principales variables que nous utiliserons. On distingue les hommes et les femmes ainsi que les différentes générations entre elles puisque les probabilités de survie sont très différentes entre sexe (seulement $26.2 \%$ des hommes survivent au moins quatre ans à 91 ans contre $44.9 \%$ des femmes) et entre générations (le taux de survie à quatre ans se situe à $95 \%$ pour les 67 ans et $80 \%$ pour les 75 ans). Les effectifs des survivants dans les différentes générations et sexes reflètent ces taux de survie puisque, par exemple, les hommes de 91 ans ne forment qu'un gros pourcent de l'échantillon. Remarquons aussi que les femmes des générations les plus jeunes ne sont pas plus nombreuses que les hommes car la sélection des personnes ayant cotisé plus de 60 trimestres fait que beaucoup de femmes ont été écartées de l'échantillon. Le nombre de trimestres de cotisation des femmes est d'ailleurs plus faible que celui des hommes (de 30 trimestres en moyenne) et l'écart ne se comble pas pour les générations les plus jeunes. L'augmentation de la participation féminine ne commence en effet que vers la fin des années 1960 et touche peu les générations que nous étudions. Ce moindre nombre de trimestres se conjugue au différentiel de salaire entre hommes et femmes pour expliquer que les droits directs versés aux femmes n'atteignent que $50 \%$ environ de ceux des hommes. En ajoutant les droits indirects dûs aux enfants, pensions de reversion, etc, cette proportion atteint $60 \%$.

Finalement, les différences de mortalité en fonction du revenu peuvent déjà se lire dans ces statistiques descriptives. Pour les hommes en effet, les droits directs pour la pension de la retraite diminuent entre les générations 18 et 30 alors que les générations les plus jeunes ont bénéficié de gains de productivité et donc de salaires plus élevés. Les taux de remplacement n'ayant pas changé sur cette période, on devrait observer le contraire. Ce phénomène correspond bien à l'idée que l'on se fait de la sélection dynamique (Cameron \& Heckman, 1998). Les plus pauvres ont une mortalité plus forte et la moyenne des pensions de retraite parmi les survivants augmente au cours du temps par un effet de composition. C'est ce que l'on va vérifier dans la sous-section suivante par un ensemble d'estimations descriptives.

\subsection{Estimation non paramétrique}

Pour décrire les données, on estime pour chaque génération et chaque sexe une régression non paramétrique de la probabilité de survie entre 1997 et 2001 sur le (logarithme du) montant des pensions en ne tenant pas compte d'autres variables explicatives. En raison 
de la malédiction de la dimensionnalité, il est en effet difficile d'estimer avec précision et de reporter des descriptions flexibles des relations multidimensionnelles entre revenu, nombre de trimestres, régions de résidence, caisses de retraite. Nous nous concentrons donc sur la seule relation entre revenu et probabilité de survie pour guider les estimations paramétriques qui tiendront compte d'autres facteurs explicatifs et dont nous présenterons les résultats dans la section suivante. La littérature suggèrant qu'une telle relation est non linéaire, nous considérerons comme seul régresseur le logarithme du revenu. Nos résultats ne démentent pas la linéarité de la probabilité de survie en fonction du logarithme du revenu.

On utilise un lissage par régression locale ${ }^{8}$ en ne tenant compte que des observations appartenant aux vingtiles intérieurs (du 2ème au 19ème) de la distribution des pensions et ceci dans un but de lisibilité. De toute façon, les bandes de confiance sont très (trop) larges aux extrêmes. L'ordre de lissage est identique pour tous les échantillons et il est choisi pour que les courbes ne soient pas trop heurtées dans les générations numériquement les plus importantes (26 et 30). On reporte aussi dans le graphique les bandes de confiance à $95 \%$. Les résultats de ces estimations sont reportés sous forme de graphiques dans les figures 1 (hommes) et 2 (femmes) pour les différentes générations. Pour les hommes, l'effet du montant est positif (dans le sens vague où une ligne horizontale ne peut pas être tracée entre les bandes de confiance) pour les hommes des générations les plus jeunes et les plus importantes, celles de 22, 26 et 30 .

Au delà de ces âges, la taille de l'échantillon devient plus petite. Les écart-types attendus sont multipliés par un facteur proportionnel à la racine de la taille de l'échantillon (voir Tableau 1) ce qui correspond aux magnitudes observées. Des effets de sélection dynamique peuvent s'être aussi mis en place. Ces derniers viennent de la présence d'hétérogénéité individuelle inobservable. On sait que cette hétérogénéité inobservable a tendance à biaiser vers le bas le taux de croissance du hasard de base (Lancaster, 1990), ici le taux de mortalité. On sait moins que cette présence d'hétérogénéité atténue l'effet des variables explicatives et ceci de manière de plus en plus forte avec le temps, sous certaines conditions il est vrai (voir annexe). L'importance de la mortalité différentielle pour les hommes, dont la magnitude sera résumée par une élasticité dans la section suivante, est donc un des résultats de cet article.

Pour les femmes au contraire, l'effet positif du revenu ne semble jamais significatif au vu des graphiques. Nous pourrions attribuer ce dernier résultat à la règle de sélection des femmes dans l'échantillon ou, ce qui est lié, à la difficulté de mesurer un revenu de cycle de vie pour elle. On ne peut pourtant pas écarter des explications structurelles. La moindre dépendance de la santé des femmes par rapport au revenu peut venir de choix personnels

\footnotetext{
${ }^{8}$ Voir Fan \& Gijbels, 1995. Nous utilisons la procédure LOWESS en Stata.
} 
d' "investissements" en santé, comme les comportements vis à vis de facteurs à risque comme le tabac et l'alcool au moins dans les générations que nous examinons. L'élasticité-revenu de ces investissements serait plus faible pour les femmes (Aliaga, 2002).

\section{Elasticités-revenu de la survie et du taux instantané de mortalité}

Pour aller plus loin, on contrôle maintenant l'effet d'autres variables explicatives comme les régions, le nombre de trimestres de cotisations, le type de caisses de retraite et la catégorie socio-professionnelle. On utilise pour cela un modèle paramétrique contraint, le modèle Probit, dont nous pouvons comparer les résultats aux résultats non-paramétriques. Nous estimons deux élasticités-revenu. Les premières sont les élasticités de la probabilité de survie à 4 ans par rapport au revenu. Cette élasticité est la seule que l'on puisse estimer à partir de nos données sans faire d'hypothèse supplémentaire. Toutefois, elle donne une information qui est difficilement comparable aux résultats d'autres études sur la mortalité différentielle. En effet, une élasticité de la probabilité de survie à quatre ans ne peut pas se comparer simplement à une élasticité de la probabilité de survie à un an ou à dix ans. Pour avoir des mesures de la mortalité différentielle plus universelles, les démographes préfèrent comparer les taux instantanés de décès. Nous estimerons donc d'autre part l'élasticité du taux de mortalité instantané par rapport au revenu. Il est clair que comme nous n'observons que la survie à 4 ans, l'estimation du taux instantané de décès impose de faire une hypothèse supplémentaire. Nous supposerons pour simplifier que cette élasticité est constante pendant les quatre ans qui séparent nos deux observations. Nous avons vu pourtant dans les régressions non paramétriques que l'élasticité du taux de mortalité par rapport au revenu est plus faible pour les plus âgés que pour les plus jeunes. Toutefois, avec une période d'observation de seulement 4 ans, qui est donc relativement courte, on peut faire abstraction de cet effet d'âge et supposer en première approximation que l'élasticité du taux instantané de mortalité par rapport au montant des pensions reste constant au cours de la période d'observation.

\subsection{Estimations Probit}

Les résultats d'estimation du modèle Probit le plus général sont reportés dans le Tableau 2 et distinguent hommes et femmes. ${ }^{9}$ Les variables explicatives sont le nombre de trimestres de cotisations, le type de caisse de retraite et des variables indicatrices de génération et de

\footnotetext{
${ }^{9}$ Nous n'avons pas pondéré nos estimations par les poids de calage reportés dans l'EIR. D'abord, la repondération n'est pas nécessaire puisque nous estimons des modèles conditionnels, ensuite les tentatives que nous avons faites montrent que les effets sont négligeables.
} 
région. De façon générale, on trouve que l'effet-revenu est positif pour les hommes et pour les femmes mais il est beaucoup plus fort pour les hommes. L'effet du revenu est significatif pour les femmes car la forme contrainte du Probit, le groupement des observations et la présence d'autres variables qui étaient omises affectent l'estimation par rapport à l'estimation nonparamétrique de la section précédente.

La nouveauté vient surtout de la présence d'autres variables. Avoir principalement cotisé aux régimes du public ou aux régimes spéciaux, ne semble pas influencer la survie par rapport aux cotisants du régime général. Par contre, la probabilité de survie décroît avec le nombre de trimestres d'assurance total pour les femmes. Ceci est un argument à l'appui de la thèse des biais de sélection pour l'échantillon féminin qui nous "empêche" de voir l'effet du revenu. En effet, celles qui ont les droits directs les plus forts auraient aussi un nombre de trimestres de cotisation plus grand. Les deux effets se compenseraient dans la régression non paramétrique.

Les effets des variables régions ne sont pas reportés dans les tableaux mais ne sont globalement significatifs que pour les hommes. Ce sont les régions minières et métallurgiques (dans le Nord et l'Est) qui ont une mortalité significativement plus élevée. En affinant le diagnostic et en isolant les différents régimes, nous avons aussi remarqué que les différences entre régions ne sont significatives que sur le sous-échantillon constitué des salariés du privé y compris les salariés agricoles.

De plus, si on sélectionne l'échantillon de plus en plus strictement en augmentant le nombre de trimestres requis pour être dans l'échantillon (au dessus de 60), les coefficients des différentes mesures du revenu augmentent. Ceci accrédite la thèse de mauvaise mesure du revenu de cycle de vie pour ceux qui ont un nombre insuffisant de trimestres de cotisation. Il y a atténuation du coefficient de cette variable vers zéro. Nous avons effectué de nombreux tests de sensibilité de ces estimations à différentes possibilité d'erreurs de spécification. Nous évaluons maintenant cette sensibilité en fonction d'un seul paramètre, l'élasticité-revenu de la probabilité de survie à quatre ans.

\subsection{Elasticités-revenu}

On estime ces élasticités à partir des résultats du modèle Probit au point moyen de l'échantillon. Dans ces estimations, on contrôle pour la région, la génération et le nombre de trimestres total de cotisation. On contrôle aussi pour le sexe lorsque l'on ne sépare pas hommes et femmes et pour le type de caisse lorsque l'on ne sépare pas les régimes ${ }^{10}$. Les estimations

\footnotetext{
${ }^{10}$ Pour les régimes spéciaux, on a omis la variable de région ; en effet, les effectifs très faibles pour les femmes entraînent que pour certaines régions, la variable dépendante n'a pas de variabilité ce qui conduit à réduire l'échantillon de façon drastique. Les variables de région ne sont pas significatives globalement pour
} 
globales des élasticités de la probabilité de survie apparaissent dans les tableaux 3 à 4 . Le Tableau 3 reporte ces élasticités quand la mesure de la pension est une mesure restreinte aux droits directs de pension de retraite. Dans le Tableau 4, nous contrastons ces résultats en utilisant le montant total de la retraite qui tient compte des droits indirects.

Les élasticités mesurées sont relativement faibles en moyenne, mais généralement significatives. Elles atteignent 9 à $10 \%$ pour les hommes. Elles sont beaucoup plus faibles pour les femmes, sauf pour les régimes spéciaux mais les effectifs féminins dans ces régimes sont très faibles. Pour les femmes, les élasticités calculées pour les droits directs ne sont significativement différentes de zéro que dans le secteur privé, ce qui confirme l'analyse non paramétrique menée dans la section précédente. Ce résultat n'est plus vérifié si l'on utilise la retraite totale. Ce montant total atteint 1.26 fois la retraite de base, avec une forte variation selon les générations, puisque pour la génération 1906, par exemple, ce rapport atteint 1.44 contre seulement 1.17 pour la génération 1930. ${ }^{11}$ A l'inverse, pour les hommes, les résultats apparaissent robustes à la mesure de la retraite (Tableau 4). Il est vrai que la retraite totale n'excède la retraite de base que de $5.3 \%$. De plus, pour ces derniers, les différences sont peu importantes entre secteur privé et fonction publique (Tableau 3).

Pour les fonctionnaires, nous pouvons utiliser une variable supplémentaire dans la base de données qui est leur indice de référence. En effet, cette variable est la mesure la plus exacte du revenu en fin de vie active dont nous disposons puisque le revenu brut est une fonction linéaire de l'indice. L'élasticité-revenu devient alors légérement plus élevée que celles que l'on a reporté dans les tableaux 3 et 4 . Ceci est conforme à l'idée que les autres mesures du revenu au cours du cycle de vie sont affectées par des erreurs qui biaisent vers le bas les coefficients du revenu. En effet, le nombre de trimestres de cotisation importe dans le calcul des pensions dans le secteur privé. Néanmoins, la magnitude de ces biais ne semble pas très importante.

\subsection{Elasticités-revenu et générations}

Pour estimer les élasticités-revenus par génération ou par âge, nous avons introduit des variables indicatrices d'âges dans les régressions que nous avons croisées avec la variable du logarithme du montant de la pension. Les coefficients et élasticités reportés ont été calculées, pour les hommes et les femmes, à la valeur moyenne des caractéristisques de l'échantillon (en particulier à la valeur moyenne du logarithme du revenu) pour chaque catégorie d'âge. Les coefficients estimés du modèle Probit sont conformes aux résultats des les régimes spéciaux ni pour les hommes, ni pour l'échantillon complet.

${ }^{11}$ Ceci est la résultante de deux effets contradictoires: la surmortalité masculine et l'augmentation de la participation féminine, faible il est vrai dans ces générations. 
régressions non-paramétriques mais sont plus précis. A partir de ces estimations, on reporte, dans le Tableau 5, les estimations de l'élasticité-revenu de la fonction de survie en fonction de la génération ou de l'âge. Pour les hommes, cette élasticité est positive, significative et constante en fonction de l'âge sauf pour la génération la plus vieille. Cela traduit deux effets qui se compensent; la décroissance de l'impact du revenu sur la survie est compensée par la décroissance de la probabilité de survie avec l'âge. Pour les femmes, les résultats sont plus complexes. L'élasticité ne devient positive et significative que dans les générations 12 et 18. Elle est alors égale à la moitié de l'élasticité revenu pour les hommes et décroît par la suite. Il faut interpréter ces résultats avec prudence à cause des effets de sélection et de notre méconnaissance du revenu familial au cours du cycle de vie.

Les troisième et quatrième colonnes du Tableau 5 donnent les élasticités du taux de mortalité instantané par rapport au revenu que l'on obtient en utilisant nos estimations sur la probabilité de survie à 4 ans et en faisant l'hypothèse que l'élasticité du taux de mortalité ne change pas au cours de la période d'observation. Nous trouvons que l'élasticité du taux instantané de mortalité par rapport au revenu va globalement en décroissant, en valeur absolue, avec l'âge. Les écarts de mortalité lié au niveau de pension semblent donc s'atténuer avec l'âge et ceci conforte l'idée de sélection dynamique (voir annexe). Finalement, nous avons aussi évalué ces élasticités-revenus, pour chaque génération et sexe, à d'autres points de l'échantillon et en particulier aux quartiles de la distribution. La distribution est approximativement symétrique, les évaluations à la médiane étant assez proches des évaluations à la moyenne. De plus, les différences entre premier et troisième quartile sont relativement faibles puisqu'elles ne valent jamais plus que la moitié, et pour la plupart des générations beaucoup moins, de l'élasticité-revenu à la médiane.

En faisant abstraction de la sélection dynamique, nous pouvons utiliser les résultats pour les hommes dans le Tableau 5 pour estimer grossièrement l'élasticité de l'espérance de vie à 60 ans. Nous supposons que le taux de mortalité est de la forme:

$$
\lambda(t)=\lambda_{0}(t) P^{\alpha(t)}
$$

où $\alpha(t)$ est une fonction en escalier qui prend les valeurs qui ressortent du Tableau 5. Plus précisément nous supposons que $\alpha(t)=-0.618$ entre 60 et 71 ans, -0.555 entre 71 et 75 ans, -0.442 entre 75 et 79 ans, -0.287 entre 79 et 85 ans, -0.182 entre 85 et 91 ans et -0.143 au delà de 91 ans. Nous utilisons la formule donnant l'espérance de vie à 60 ans:

$$
E_{60}=\int_{60}^{+\infty} \exp \left(-\int_{60}^{t} \lambda(\tau) d \tau\right) d t
$$

pour calculer l'élasticité revenu de l'espérance de vie lorsque les taux de mortalité corre- 
spondent au taux moyens observés en $1997 .{ }^{12}$ Nous trouvons une élasticité de l'espérance de vie à 60 par rapport au niveau des pensions de l'ordre de 0.18. En extrapolant, cela indiquerait qu'un individu touchant une pension deux fois plus élevée qu'un autre individu aurait aussi une espérance de vie à 60 ans de $18 \%$ supérieur. Ces calculs sont bien sûr à prendre avec beaucoup de précaution, puisqu'ils résultent d'une extrapolation grossière et qu'ils n'indiquent pas non plus d'intervalle de confiance, mais ils semblent indiquer que l'on mesure des effets comparables, par leur ordre de grandeur, à ceux estimés par Desplanques (1991, 1993) pour la mortalité différentielle selon la CSP.

\subsection{Revenu et catégories socio-professionnelles}

On revient ici sur le débat des effets relatifs des professions et du revenu. Est-ce le revenu qui affecte directement la probabilité de survie ou le fait-il à travers la profession exercée? On ne peut apporter des éléments de réponse très précis à ce débat puisque nous ne disposons que d'une mesure très approximative des professions par leur regroupement en ouvriers qualifiés, ouvriers spécialisés, employés, professions intermédiaires et cadres. Cette information est enregistrée au moment de la retraite. Comme elle est absente des renseignements fournis par les caisses de retraite, nous avons utilisé les DADS et les données UNEDIC pour l'obtenir. Elle n'est donc disponible que pour les salariés du secteur privé ayant connu au moins un épisode d'emploi ou de chômage entre 1985 et la date de liquidation. Nous sommes ainsi obligés de nous restreindre aux générations, 1926 et 1930, puisque, pour la plupart des individus des générations plus anciennes, nous n'avons pas d'informations sur leurs professions. Le Tableau 6 propose une estimation pour les hommes, de l'effet sur la probabilité de survie du montant de la retraite en contrôlant pour ces catégories. La catégorie de référence utilisée est « Cadres et chefs d'entreprise ».

Les effets de la retraite restent positifs et significatifs que ce soit en utilisant la somme des avantages principaux de droit direct ou le montant total de la retraite. L'élasticité est néanmoins plus faible et égale à environ 0.05. Par ailleurs, la probabilité de survie est plus faible pour toutes les catégories par rapport aux cadres, mais l'effet n'est significatif que pour les ouvriers qualifiés. Mais il est vrai que si on omet la variable de pension de la régression, comme on le reporte dans la deuxième colonne, les effets des catégories doublent en magnitude et deviennent significatifs. La forte corrélation par "construction" entre catégories et revenus en est responsable mais il est difficile d'aller plus loin que ce constat.

\footnotetext{
${ }^{12}$ Ces taux proviennent du Human Mortality Database, http://www.mortality.org
} 


\section{Mortalité différentielle et systèmes de retraite}

L'estimation de l'élasticité du taux instantané de mortalité par rapport au revenu est bien sûr très intéressante pour le démographe et toute personne intéressée par les déterminants de la mortalité. Elle pourrait par exemple être utilisée pour la définition de politiques publiques de santé. Mais cette information est également utile pour traiter des questions relatives à la gestion des systèmes de retraite. C'est ce dernier point que nous développons ci dessous, en examinant deux points particuliers : l'élasticité de l' "équivalent-capital" retraite par rapport au montant de pension de retraite, ce qui permet d'évaluer l'ampleur de l'effet antiredistributif de la mortalité différentielle, d'une part, et le lien entre mortalité différentielle et dates de départ à la retraite, d'autre part. Notre objectif est par là de montrer quels sont les ordres de grandeur des phénomènes auxquels on peut s'attendre au vu des élasticités estimées. Une approche plus détaillée des divers déterminants des caractères redistributifs ou anti-redistributifs du système français de retraite est disponible dans Walraet et Vincent (2003).

Pour mener à bien ces exercices nous supposerons que l'élasticité-revenu du taux instantané de mortalité est une constante $\alpha$ indépendante de l'âge. Comme nous ne pouvons pas traiter de façon rigoureuse dans cet article du problème de la sélection dynamique, cette hypothèse est difficile à tester. Elle permet toutefois de faire des calculs relativement simples qui pourront être utilisés pour donner une fourchette des effets auxquels on peut s'attendre.

\subsection{Elasticité de l" "équivalent-capital" retraite par rapport au mon- tant de pension de retraite.}

Nos estimations ont montré que les individus qui touchent une pension élevée vivent en moyenne plus longtemps. De fait, si un individu touche une pension mensuelle deux fois plus élevée qu'un autre individu, nous savons, du fait de la mortalité différentielle, que la somme totale des pensions qu'il touchera en moyenne sera plus de deux fois plus élevée que celle que touchera l'autre individu. Pour donner une idée de l'ampleur de ce phénomène nous calculons ci-dessous l'élasticité de l' "équivalent-capital" retraite par rapport au montant de pension de retraite.

Précisément supposons que les marchés intertemporels sont parfaits et que le taux d'intérêt est égal à $r$. Les individus sont alors indifférents entre recevoir une pension $P$ entre le moment où ils liquident leur droit à la retraite, $a_{L}$, et leur décès, ou recevoir au moment de la liquidation un capital donné par:

$$
K\left(a_{L}, P\right)=P \int_{a_{L}}^{+\infty} s_{a_{L}, P}(t) e^{-r\left(t-a_{L}\right)} d t
$$


où $s_{a_{L}, P}(t)$ est la fonction de survie à partir du moment $a_{L}$ (i.e. $s_{a_{L}, P}\left(a_{L}\right)=1$ ) pour un niveau de pension $P$.

Dans le cas où le taux instantané de décès est donné par $\lambda(t)=P^{\alpha} \lambda_{0}(t)$, où $\lambda_{0}(\tau)$ est le taux de hasard de base, on a (voir annexe):

$$
\log \left(s_{a_{L}, P}(t)\right)=-\exp (\alpha \log (P)) \int_{a_{L}}^{t} \lambda_{0}(\tau) d \tau
$$

Par un calcul direct, on obtient:

$$
\frac{\partial s_{a_{L}, P}(t)}{\partial \log P}=-\alpha s_{a_{L}, P}(t) \exp (\alpha \log (P)) \int_{a_{L}}^{t} \lambda_{0}(\tau) d \tau=\alpha s_{a_{L}, P}(t) \log \left(s_{a_{L}, P}(t)\right)
$$

et donc

$$
\frac{\partial \log K}{\partial \log P}=1+\alpha\left[\frac{\int_{a_{L}}^{+\infty} s_{a_{L}, P}(t) \log \left(s_{a_{L}, P}(t)\right) e^{-r\left(t-a_{L}\right)} d t}{\int_{a_{L}}^{+\infty} s_{a_{L}, P}(t) e^{-r\left(t-a_{L}\right)} d t}\right] .
$$

Cette élasticité dépend donc du taux d'actualisation, de l'âge à la liquidation et de la mortalité des individus. Toutefois on peut l'estimer de façon approximative en prenant pour $\alpha$ des valeurs cohérentes avec celles estimées auparavant, $a_{L}=60$ et en supposant que $s_{a_{L}, P}(t)$ est proche de la fonction de survie que l'on obtient en utilisant les taux de mortalité observés pour les hommes en France en 1997.

Les résultats, pour différentes valeur de $\alpha$ et de $r$, sont reportés dans le Tableau 7 . Nous utilisons deux valeurs différentes pour $\alpha$, proches mais non égales des estimations, pour montrer la sensibilité des calculs simplifiés que nous menons. Comme on le voit, on trouve des élasticités qui sont supérieures à 1 tout en restant assez proches de 1. La mortalité différentielle liée au niveau des pensions engendre donc une redistribution financière régressive. Doubler le revenu revient à faire plus que doubler l'équivalent capital de 8 à $17 \%$ dans les scénarios centraux. Cet impact est loin d'être négligeable et doit être mis en regard des autres dimensions redistributives du mode de calcul des pensions. Par exemple, en reprenant le Rapport de stratégie nationale sur les pensions rédigé pour l'Union Européenne par la France (2002), ${ }^{13}$ on trouve qu'un doublement approximatif du revenu entre les tranches $7500-10000 \mathrm{~F}$ et $15000-20000 \mathrm{~F}$ fait passer le taux de remplacement de $91 \%$ à $72 \%$ (page 8 de l'annexe). L'élasticité-revenu correspondant à ce transfert est donc de 0.8. L'effet de la mortalité différentielle annule entre $1 / 4$ et plus de la moitié de cette redistribution $\left(0.8^{*} 1.08\right.$ ou $0.8^{*} 1.17$ ) suivant nos hypothèses de tendance centrales extraites du Tableau 5. Examiner les taux de remplacement pourrait donc exagèrer les effets redistributifs du système de retraite en France. Cet effet anti-redistributif semble plus fort que celui que Walraet et Vincent (2003) trouve par microsimulation mais les résultats sont difficiles à comparer puisque

\footnotetext{
${ }^{13}$ http://europa.eu.int/comm/employment_social/soc-prot/pensions/fr_pensionreport_fr.pdf
} 
ces auteurs, d'une part, n'utilisent pas d'élasticités-revenus comme nous mais une approche par déciles et par taux de rendement interne, d'autre part, utilisent la mortalité différentielle entre groupes d'éducation et non de revenus. La conclusion générale reste pourtant la même. La mortalité différentielle est anti-redistributive mais le système de retraites, considéré dans sa généralité, reste redistributif.

\subsection{Mortalité différentielle et incitations au départ à la retraite.}

La mortalité différentielle peut aussi affecter les incitations qu'ont les individus à liquider leur retraite à un âge donné. Le législateur peut donc vouloir utiliser l'information qu'il acquiert sur la mortalité des individus via leur revenu au moment du départ en retraite pour définir le mode de calcul des pensions de retraites qui sera cohérent avec les incitations qu'il souhaite mettre en place (Blanchet et Caussat, 2001).

Afin d'illustrer l'impact de la mortalité différentielle, imaginons un scénario où le planificateur souhaite supprimer les effets incitatifs et s'orienter vers un système actuariellement neutre. On considère donc le cas où le planificateur observe un revenu de référence $R$ et où il sait que le taux de mortalité à l'âge $t$ d'un individu ayant ce revenu de référence est donné par $\lambda(t)=R^{\alpha} \lambda_{0}(t) .{ }^{14}$ Le problème du planificateur qui cherche à définir un mode de calcul des pensions actuariellement neutre consiste à déterminer le taux de remplacement $\rho_{R}\left(a_{L}\right)$ tel que pour une pension $\rho_{R}\left(a_{L}\right) R$ l'équivalent en capital des pensions auquel on a soustrait l'équivalent des cotisations versées soit nul et ceci pour tout âge à la liquidation, $a_{L}$. En d'autres termes et en supposant le taux de cotisation constant le planificateur souhaite définir $\rho_{R}\left(a_{L}\right)$ de telle sorte que, pour tout $a_{L}$ :

$$
\rho_{R}\left(a_{L}\right) R \int_{a_{L}}^{+\infty} s_{R}(t) e^{-r t} d t=\tau R \int_{0}^{a_{L}} s_{R}(t) e^{-r t} d t
$$

où $s_{R}(t)$ est la fonction de survie à partir de la date 0 pour un niveau de revenu $R$. En dérivant cette expression par rapport à l'âge de liquidation, on obtient:

$$
\rho_{R}^{\prime}\left(a_{L}\right) R \int_{a_{L}}^{+\infty} s_{R}(t) e^{-r t} d t-\rho_{R}\left(a_{L}\right) R s_{R}\left(a_{L}\right) e^{-r a_{L}}=\tau R s_{R}\left(a_{L}\right) e^{-r a_{L}}
$$

Une solution de cette équation différentielle est:

$$
\rho_{R}\left(a_{L}\right)=-\tau+C \exp \left(\int_{0}^{a_{L}} \beta_{R}(t) d t\right)
$$

\footnotetext{
${ }^{14}$ On suppose ici pour simplifier que la mortalité différentielle selon le niveau des pensions que l'on a observée traduit en fait une mortalité différentielle selon le revenu de référence. Tant qu'il y a une relation univoque entre pension et revenu, notre analyse est correcte à condition de corriger les élasticités-revenus des aspects redistributifs exposés plus haut.
} 
où le facteur $\beta$ indique dans quelle proportion le montant des pensions doit augmenter en fonction de l'âge à la liquidation pour que le barème soit actuariellement neutre "horizontalement" c'est à dire pour un certain montant de revenu $R$. La neutralité actuarielle "verticale" nous permettrait par exemple de déterminer $C$ en fonction de $R$. Nous le supposons ici constant.

De l'équation 2 il vient:

$$
\beta_{R}\left(a_{L}\right)=\frac{1}{\int_{a_{L}}^{+\infty} s_{a_{L}, R}(t) e^{-r\left(t-a_{L}\right)} d t}
$$

La question est de savoir dans quelle mesure $\beta$ dépend de $R$. Pour cela on peut calculer l'élasticité de $\beta$ par rapport à $R$. On obtient :

$$
\frac{\partial \log (\beta)}{\partial \log (R)}=-\alpha\left[\frac{\int_{a_{L}}^{+\infty} s_{a_{L}, R}(t) \log \left(s_{a_{L}, R}(t)\right) e^{-r\left(t-a_{L}\right)} d t}{\int_{a_{L}}^{+\infty} s_{a_{L}, R}(t) e^{-r\left(t-a_{L}\right)} d t}\right]
$$

Ce résultat dépend donc aussi de $r$ et de la fonction de survie. En fait, à cause de nos hypothèses et en particulier celle de taux de cotisation constant, cette quantité peut se dériver directement de l'élasticité de l' "équivalent-capital" retraite donnée par l'équation (1). Elle est égale à 1 moins cette dernière élasticité et ses valeurs se dérivent donc directement du Tableau 9. Or, le coefficient $\beta_{R}$ s'interprète comme le taux d'accroissement du taux de remplacement (en d'autres termes la valeur relative du point de retraite supplémentaire) qui doit être mis en place pour aboutir à un système actuariellement neutre lorsque $\tau$, le taux de cotisation, est négligeable par rapport à $\rho_{R}$, le taux de remplacement. On voit donc que, lorsque l'on double le revenu ce taux d'accroissement devrait diminuer de 8 à 17 \%. Là aussi, les effets de la mortalité différentielle selon le niveau de revenu va générer des ajustements non négligeables mais modérés.

\section{Conclusion}

En utilisant les données de l'échantillon inter-régime des retraités nous avons mesuré la mortalité différentielle en fonction du niveau des pensions. Cette information est nouvelle en France, puisqu'à notre connaissance aucune étude n'avait étudié précisément le lien entre mortalité et revenu.

Nos résultats indiquent une corrélation négative et significative entre mortalité et revenu pour les hommes. Cet effet est robuste et ressort d'estimations non paramétriques. Des estimations paramétriques nous permettent de tenir compte d'autres déterminants de la mortalité et du calcul de la pension. Nous trouvons que l'élasticité du taux de mortalité au montant de pension est de l'ordre de -0.55 entre 71 et 75 ans et de -0.18 entre 85 et 91 ans 
pour les hommes. L'élasticité de l'espérance de vie à 60 ans serait de l'ordre de 0.18. Ces résultats sont d'un ordre de grandeur comparable à ceux obtenus par les études documentant la mortalité différentielle selon la CSP. Les résultats pour les femmes ne sont significatifs que dans certaines spécifications mais cela est sans doute dû au fait que les données de l'EIR sont loin d'être représentatives de la population féminine et que les données de revenus reflétent imparfaitement sans doute les revenus familiaux dont elles ont pu disposer au cours du cycle de vie.

Les données des caisses de retraite ne permettent pas de dissocier entre l'effet des revenus et l'effet des professions. L'échantillon interrégimes des retraités par son appariement aux données DADS et aux données de l'UNEDIC, permet de contrôler par la CSP, sans que l'on puisse toutefois avoir un contrôle très fin au niveau de la profession. Nos estimations indiquent, qu'à même CSP, il reste, pour les hommes, une mortalité différentielle significative selon le niveau des pensions. Nos résultats sur la mortalité différentielle selon le revenu viennent donc compléter ceux déjà bien connus sur la mortalité différentielle par CSP.

A titre d'exercice nous avons étudié quel pouvait être l'impact de la mortalité différentielle pour la gestion des systèmes de retraites. Nous avons estimé d'une part la redistribution financière qui résulte du fait que ceux qui touchent des pensions plus élevées sont en moyenne aussi ceux qui vivent le plus longtemps. Nous avons calculé d'autre part les taux de remplacement qu'il faudrait mettre en place pour s'orienter vers un mode de calcul du montant des pensions actuariellement neutre en dépit de cette mortalité différentielle. Dans les deux cas nous sommes arrivés à des effets qui ne sont pas négligeables. La mortalité différentielle liée au niveau des pensions est donc à même d'amener à réviser profondément la réflexion sur la gestion des systèmes de retraite et sur leurs vertus redistributives.

Soulignons pour terminer que si nous avons discuté des redistributions financières liées à la mortalité différentielle, nous n'avons pas abordé la question des effets de la mortalité différentielle en terme de bien être. Cela ne peut être fait sans aborder la question difficile de la valeur de la vie. Cette absence et notre discussion qui se focalise sur les systèmes de retraites ne doivent pas faire oublier pour autant que la mortalité différentielle est une source primordiale d'inégalité. L'inégalité ne réside pas tant dans le fait que les plus aisés touchent leur pension plus longtemps que les autres, mais tout simplement dans le fait qu'ils vivent en moyenne plus longtemps que les autres. 


\section{Bibliographie}

Adams,P., M.D., Hurd, D., McFadden, A., Merrill and T., Ribeiro, 2003, "Healthy, Wealthy and Wise? Tets for Direct Causal Paths between Health and Socioeconomic Status", Journal of Econometrics, 112:3-56.

Aliaga, C., 2002, "Les femmes plus attentives à leur santé que les hommes", Insee première, $\mathrm{n}^{\circ} 869$.

Attanasio, O. et C. Emmerson, 2003, "Mortality, Health Status, and Wealth", Journal of the European Economic Association, 1:821-50.

Bertillon J. 1889. De la morbidité et de la mortalité professionnelle (étude accompagnée d'une nouvelle table de mortalité par professions, d'après les Annuaires statistiques de Paris, 1885-89). Annuaire statistique de la ville de Paris 186-236.

Blanchet, D., et L.,Caussat, 2001, "Le libre-choix de l'âge de la retraite: approches économiques", in D.Taddei, Retraites Choisies et Progressives, Conseil d'Analyse Economique, 21: $233-50$

Bommier, A., T., Magnac, M., Roger, 2003, "Le marché du travail à l'approche de la retraite en France entre 1982 et 1999: évolutions et évaluations", Revue Française d'Economie, XVIII:23-82.

Cameron, S.V., et J.J., Heckman, 1998, "Life-Cycle Schooling and Dynamic Selection Bias", Journal of Political Economy, 106:262-333.

Deaton, A.S., et C.H., Paxson, 1998, "Aging and Inequality in Income and Health", American Economic Review, 88:248-53.

Desplanques G., 1973, "A 35 ans les instituteurs ont encore 41 ans à vivre, les manoeuvres 34 seulement" Economie et Statistique 49:3-19..

Desplanques G. 1991. Les cadres vivent plus vieux. INSEE Première.158:1-4.

Desplanques G. 1993. L'inégalité sociale devant la mort. In INSEE. La société française : données sociales 1993. Paris: INSEE, 251-258.

DREES, 1996, "Des retraites aux retraités : l'échantillon interrégimes de retraités opération 1993, document méthodologique" Documents statistiques $\mathrm{n}^{\circ} 255$.

Fan, J., et I., Gijbels, 1996, Local Polynomial Modelling and Its Applications, Chapman \& Hall:London.

Febvay M, Aubenque M. 1957. La mortalité par catégorie socio-professionnelle. Etudes statistiques (supplément trimestriel du Bulletin Mensuel de Statistique) 3:39-44.

Huber M. 1912, Mortalité suivant la profession, d'après les décès enregistrés en France en 1907 et 1908. Bulletin de la statistique générale de la France, fascicule IV, juillet 1912, 402-439.

Jusot, F., 2003, "Mortalité, Revenus et Inégalités de Revenus", document présenté au congrès de l'AFSE.

Lancaster, A., 1990, The Econometric Analysis of Transition Data, Cambridge University Press: Cambridge.

Ledermann S. 1960. Estimation de l'espérance de vie à la naissance par catégorie professionnelle en France. Population (1):127-131.

Marmot, M.G., G.D., Smith, S., Stansfeld, C., Patel, F., North, J., Head, I.White, E., Brunner, et A., Feeny, 1991, "Health Inequalities among British Cicil Servants: the Whitehall II study, Lancet, 337:1387-1393. 
Mesrine A. 1999. Les différences de mortalité par milieu social restent fortes. In INSEE. La société française : données sociales 1999. Paris: INSEE, 228-235.

Mustard CA, Derksen S, Berthelot JM, Wolfson M, Roos LL. 1997. Age-specific education and income gradients in morbidity and mortality in a Canadian province. Social Science and Medicine 45(3):383-97.

Smith, J.P., 1999, "Healthy Bodies and Thick Wallets: The Dual Relation Between Health and Economic Status", Journal of Economic Perspectives, 13:145-66.

Snyder, S.E., et W.N, Evans, 2002, "The Impact of Income and Mortality: Evidence from the Social Security Notch", working paper, University of Maryland.

Walraet, E. et A., Vincent, 2003, "La redistribution intragénérationnelle dans le système de retraite des salariés du privé: une approche par microsimulation", Economie et Statistique, 366:31-61. 


\section{Annexe}

On répéte ici des arguments proposées dans Lancaster (1990) par exemple pour montrer comment on calcule l'élasticité-revenu du taux de mortalité et pour justifier la baisse de ces estimations au fur et à mesure que l'âge croit.

Les démographes s'intéressent au hasard ou taux instantané de décès. On supposera que l'élasticité de ce hasard par rapport au revenu est constante et égale à $\alpha$ ce qui donne:

$$
\lambda(t)=\exp (\alpha \log P) \lambda_{0}(t)
$$

où $\lambda_{0}(t)$ est le hasard de base et où $P$ est le niveau des pensions. La fonction de survie en $t$ s'obtient par intégration de cette fonction

$$
s_{P}(t)=\exp \left(-\exp (\alpha \log P) \int_{0}^{t} \lambda_{0}(\tau) d \tau\right)
$$

où $\left.\int_{0}^{t} \lambda_{0}(\tau) d \tau\right)=\Lambda_{0}(t)$ est le hasard intégré. On a donc:

$$
\log \left(-\log \left(s_{P}(t)\right)\right)=\alpha \log P+\log \Lambda_{0}(t)
$$

Si on introduit maintenant un terme d'hétérogénéité inobservable, $v$, le hasard devient:

$$
\lambda(t, v)=v \exp (\alpha \log P) \lambda_{0}(t)
$$

qui, en l'intégrant dans la population survivant en $t$, donne:

$$
\lambda(t)=E_{T \geq t} \lambda(t, v)=E(v \mid T \geq t) \exp (\alpha \log P) \lambda_{0}(t)
$$

où le terme $E(v \mid T \geq t)$ décrit le processus de sélection dynamique. On écrit:

$$
E(v \mid T \geq t)=\frac{E(v \mathbf{1}\{T \geq t\})}{\operatorname{Pr}(T \geq t)}=\frac{\int v s_{P}(t \mid v) f(v) d v}{\int s_{P}(t \mid v) f(v) d v}
$$

où $f(v)$ est la densité dans la population de départ de $v$ supposé indépendante de $P$. La sélection dynamique a pour objet de créer de la dépendance entre $v$ et $P$ au fur et à mesure du temps. En effet, d'après l'adaptation de (3) à la présence d'hétérogénéité:

$$
\frac{\partial}{\partial \log P} s_{P}(t \mid v)=-\alpha v \exp (\alpha \log P) \Lambda_{0}(t) S(t \mid v)
$$

Ainsi:

$$
\begin{aligned}
\frac{\partial}{\partial \log P} E(v & \mid T \geq t)= \\
= & -\alpha \exp (\alpha \log P) \Lambda_{0}(t) \frac{\int v^{2} s_{P}(t \mid v) f(v) d v \cdot \operatorname{Pr}(T \geq t)-\left(\int v s_{P}(t \mid v) f(v) d v\right)^{2}}{\operatorname{Pr}(T \geq t)^{2}} \\
= & -\alpha \exp (\alpha \log P) \Lambda_{0}(t) V(v \mid T \geq t)<0
\end{aligned}
$$


L'élasticité du taux de mortalité par rapport au niveau des pensions est donc atténuée vers zéro (sauf en $t=0$ ) puisque:

$$
\frac{\partial \log \lambda(t)}{\partial \log P}=\alpha\left(1-\Lambda_{0}(t) \exp (\alpha \log P) \frac{V(v \mid T \geq t)}{E(v \mid T \geq t)}\right)
$$

L'évolution au cours du temps dépend de la forme de la loi de $v$. Néanmoins, on peut penser que le rapport $V(v \mid T \geq t) / E(v \mid T \geq t)$ est un terme dont l'évolution au cours du temps est du deuxième ordre par rapport à celle du hasard intégré $\Lambda_{0}(t)$ qui augmente à un rythme accéléré si le taux de mortalité augmente au cours du temps. 
Tableau 1: Statistiques descriptives de l'échantillon

\begin{tabular}{rcccccr}
\hline & Génération & $\begin{array}{c}\text { Probabilité de } \\
\text { survie entre 1997 } \\
\text { et 2001 }\end{array}$ & $\begin{array}{c}\text { Avantages } \\
\text { principaux, } \\
\text { droits } \\
\text { directs }\end{array}$ & $\begin{array}{c}\text { Retraite } \\
\text { totale }\end{array}$ & $\begin{array}{c}\text { Nombre de } \\
\text { trimestres } \\
\text { total }\end{array}$ & Effectif \\
\hline $\mathbf{H}$ & 1906 & 26.2 & 8555.2 & 9265.3 & 136.8 & 279 \\
$\mathbf{O}$ & 1912 & 53.5 & 9553.6 & 10154.8 & 144.3 & 112 \\
$\mathbf{M}$ & 1918 & 72.4 & 10123.3 & 10727.8 & 154.8 & 1399 \\
$\mathbf{M}$ & 1922 & 80.7 & 10101.3 & 10654.2 & 164.5 & 2965 \\
$\mathbf{E}$ & 1926 & 85.1 & 9905.3 & 10403.5 & 165.6 & 3741 \\
$\mathbf{S}$ & 1930 & 89.3 & 9612.6 & 10064.0 & 166.4 & 2845 \\
& Total & 79.4 & 9847.4 & 10374.1 & 161.7 & 12341 \\
$\mathbf{F}$ & 1906 & & & & & \\
$\mathbf{E}$ & 1912 & 44.9 & 4164.0 & 5998.6 & 110.0 & 541 \\
$\mathbf{M}$ & 1918 & 68.7 & 4515.1 & 6381.7 & 118.6 & 1525 \\
$\mathbf{M}$ & 1922 & 83.1 & 4662.3 & 6289.7 & 124.3 & 1612 \\
$\mathbf{E}$ & 1926 & 89.7 & 4914.5 & 6274.0 & 131.2 & 3165 \\
$\mathbf{S}$ & 1930 & 94.0 & 5093.1 & 6118.5 & 136.9 & 3710 \\
& Total & 95.6 & 5061.3 & 5897.5 & 138.2 & 2710 \\
\hline & & 87.1 & 4887.3 & 6156.6 & 131.1 & 13263 \\
\hline
\end{tabular}

Tableau 2: Estimations de la probabilité de survie: modèle de base (droits directs de pension)

\begin{tabular}{|c|c|c|c|c|c|c|c|c|}
\hline & \multicolumn{4}{|c|}{ Hommes } & \multicolumn{4}{|c|}{ Femmes } \\
\hline Logarithme de la Pension & 0.081 & $(10.6)$ & .076 & $(8.8)$ & 0.023 & $(5.2)$ & .016 & $(2.5)$ \\
\hline Nombre de trimestres & - & & $-8.5 .10^{-5}$ & $(-0.5)$ & - & & $-1.9 .10^{-4}$ & $(-2.0)$ \\
\hline Secteur public et coll. locales & - & & $-4.6 .10^{-3}$ & $(-0.5)$ & - & & -0.012 & $(-1.2)$ \\
\hline Régimes spéciaux & - & & $-4.0 \cdot 10^{-3}$ & $(-0.3)$ & - & & -0.011 & $(-0.5)$ \\
\hline Log Vraisemblance & \multicolumn{2}{|c|}{-6217.67} & \multicolumn{2}{|c|}{-5676.77} & -509 & 7.39 & \multicolumn{2}{|c|}{-4421.57} \\
\hline Pseudo- $R^{2}$ & & $\%$ & 9.5 & & 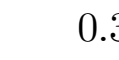 & & 13.5 & \\
\hline Nombre d'observations & \multicolumn{4}{|c|}{12341} & \multicolumn{4}{|c|}{13263} \\
\hline
\end{tabular}

Notes : Estimation Probit. Entre parenthèses figurent les statistiques de Student.

Le tableau présente les effets marginaux. Pour les indicatrices les effets marginaux sont calculés comme pour les variables continues (produit du coefficient par la densité calculée à la moyenne de l'échantillon). Dans les estimations des colonnes 2 et 4, on inclue aussi des variables indicatrices de la génération et des zones géographiques (région de résidence). 
Tableau 3: Elasticités de survie aux droits directs de pension de retraite.

\begin{tabular}{lllll}
\hline & Ensemble & $(1)$ & $(2)$ & $(3)$ \\
\hline \multirow{3}{*}{ Hommes } & 0.094 & 0.096 & 0.108 & 0.059 \\
& $(0.011)$ & $(0.012)$ & $(0.031)$ & $(0.045)$ \\
Femmes & 0.019 & 0.021 & -0.026 & 0.147 \\
& $(0.007)$ & $(0.008)$ & $(0.029)$ & $(0.097)$ \\
Ensemble & 0.047 & 0.051 & 0.046 & 0.070 \\
& $(0.006)$ & $(0.007)$ & $(0.020)$ & $(0.040)$ \\
& & & & \\
\hline Effectifs & 25604 & 19818 & 4289 & 1497 \\
\hline
\end{tabular}

$\underline{\text { Notes }}:(1)=$ CNAV et MSA; $(2)=$ Fonction publique et collectivités territoriales; $(3)=$ Régimes spéciaux (indicatrices de région omises dans ce cas). Ecart-types entre parenthèses. L'élasticité est calculée au point moyen de l'échantillon et son écart-type est dérivé par la méthode delta.

Tableau 4: Elasticités de survie au montant total de pension de retraite.

\begin{tabular}{lllll}
\hline & Ensemble & $(1)$ & $(2)$ & $(3)$ \\
\hline \multirow{3}{*}{ Hommes } & 0.089 & 0.093 & 0.094 & 0.042 \\
& $(0.011)$ & $(0.012)$ & $(0.031)$ & $(0.044)$ \\
Femmes & -0.001 & -0.002 & -0.013 & 0.158 \\
& $(0.007)$ & $(0.007)$ & $(0.023)$ & $(0.079)$ \\
Ensemble & 0.035 & 0.035 & 0.040 & 0.061 \\
& $(0.006)$ & $(0.006)$ & $(0.019)$ & $(0.039)$ \\
\hline
\end{tabular}

Note : Notes: $(1)=$ CNAV et MSA; $(2)=$ Fonction publique et collectivités territoriales; $(3)=$ Régimes spéciaux (indicatrices de région omises dans ce cas). Ecart-types entre parenthèses. L'élasticité est calculée au point moyen de l'échantillon et son écart-type est dérivé par la méthode delta. 
Tableau 5: Elasticités aux droits directs de pension de retraite, par âge et sexe

\begin{tabular}{|c|c|c|c|c|}
\hline & \multicolumn{2}{|c|}{ Elasticités de survie } & \multicolumn{2}{|c|}{ Elasticités taux de mortalité } \\
\hline & Hommes & Femmes & Hommes & Femmes \\
\hline Génération 30 - 67 ans & $\begin{array}{c}0.085 \\
(0.022)\end{array}$ & $\begin{array}{c}0.003 \\
(0.006)\end{array}$ & $\begin{array}{l}-0.618 \\
(0.131)\end{array}$ & $\begin{array}{l}-0.061 \\
(0.153)\end{array}$ \\
\hline Génération 26 - 71 ans & $\begin{array}{c}0.110 \\
(0.021)\end{array}$ & $\begin{array}{c}0.006 \\
(0.006)\end{array}$ & $\begin{array}{r}-0.555 \\
(0.088)\end{array}$ & $\begin{array}{r}-0.113 \\
(0.119)\end{array}$ \\
\hline Génération 22 - 75 ans & $\begin{array}{c}0.113 \\
(0.025)\end{array}$ & $\begin{array}{l}0.0190 \\
(0.009)\end{array}$ & $\begin{array}{r}-0.442 \\
(0.085)\end{array}$ & $\begin{array}{r}-0.189 \\
(0.105)\end{array}$ \\
\hline Génération 18 - 79 ans & $\begin{array}{c}0.104 \\
(0.041)\end{array}$ & $\begin{array}{c}0.047 \\
(0.016)\end{array}$ & $\begin{array}{c}-0.287 \\
(0.102)\end{array}$ & $\begin{array}{l}-0.287 \\
(0.109)\end{array}$ \\
\hline Génération 12 - 85 ans & $\begin{array}{c}0.122 \\
(0.061)\end{array}$ & $\begin{array}{c}0.068 \\
(0.027)\end{array}$ & $\begin{array}{r}-0.182 \\
(0.085)\end{array}$ & $\begin{array}{r}-0.195 \\
(0.084)\end{array}$ \\
\hline Génération 06 - 91 ans & $\begin{array}{c}0.202 \\
(0.197)\end{array}$ & $\begin{array}{c}0.009 \\
(0.079)\end{array}$ & $\begin{array}{r}-0.143 \\
(0.134)\end{array}$ & $\begin{array}{r}-0.012 \\
(0.097)\end{array}$ \\
\hline
\end{tabular}

Note : Notes: Ecart-types entre parenthèses. L'élasticité est calculée au point moyen de l'échantillon et son écart-type est dérivé par la méthode delta. 
Tableau 6: Catégories socio-professionnelles et revenus

\begin{tabular}{|c|c|c|c|c|}
\hline \multicolumn{5}{|l|}{ Hommes } \\
\hline Log de la pension totale & 0.043 & $(2.84)$ & - & \\
\hline Professions Intermédiaires & -0.014 & $(-0.73)$ & -0.030 & $(-1.63)$ \\
\hline Employés & -0.012 & $(-0.61)$ & -0.040 & $(2.05)$ \\
\hline Ouvriers Qualifiés & -0.041 & $(-2.12)$ & -0.073 & $(4.44)$ \\
\hline Ouvriers Non Qualifiés & -0.039 & $(-1.37)$ & -0.079 & $(2.86)$ \\
\hline Génération 1926 & -0.049 & $(-4.74)$ & -0.048 & $(4.63)$ \\
\hline Nombre d'observations & \multicolumn{2}{|c|}{4104} & \multicolumn{2}{|c|}{4104} \\
\hline Log-Vraisemblance & \multicolumn{2}{|c|}{-1507.10} & \multicolumn{2}{|c|}{-1511.22} \\
\hline
\end{tabular}

Note: Statistiques de Student entre parenthèses. La modalité de référence pour les CSP est la catégorie des cadres.

Tableau 7: Elasticité de l'équivalent capital au montant mensuel de la pension.

\begin{tabular}{ccc}
\hline & \multicolumn{2}{c}{ Elasticité du taux de mortalité } \\
\hline Taux d'intérêt & $-0,3$ & $-0,5$ \\
& & \\
\hline & \multicolumn{2}{c}{ Elasticité de l'équivalent capital } \\
\hline 0.00 & 1.124 & 1.206 \\
0.01 & 1.112 & 1.187 \\
0.02 & 1.101 & 1.169 \\
0.03 & 1.092 & 1.153 \\
0.04 & 1.084 & 1.139 \\
0.05 & 1.076 & 1.127 \\
0.06 & 1.069 & 1.115 \\
\hline
\end{tabular}

Note: Pour les modalités du calcul, voir le texte. 

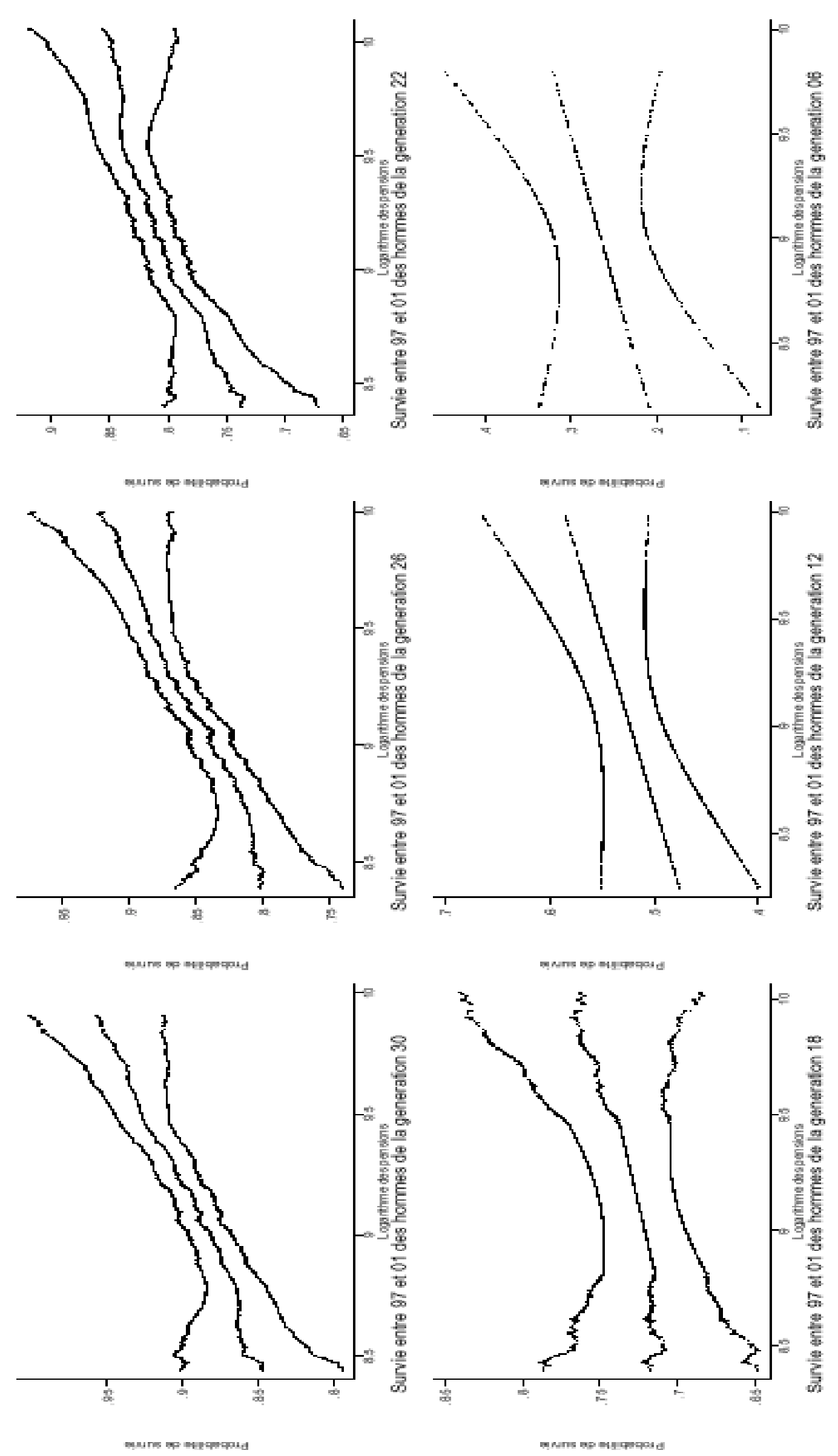

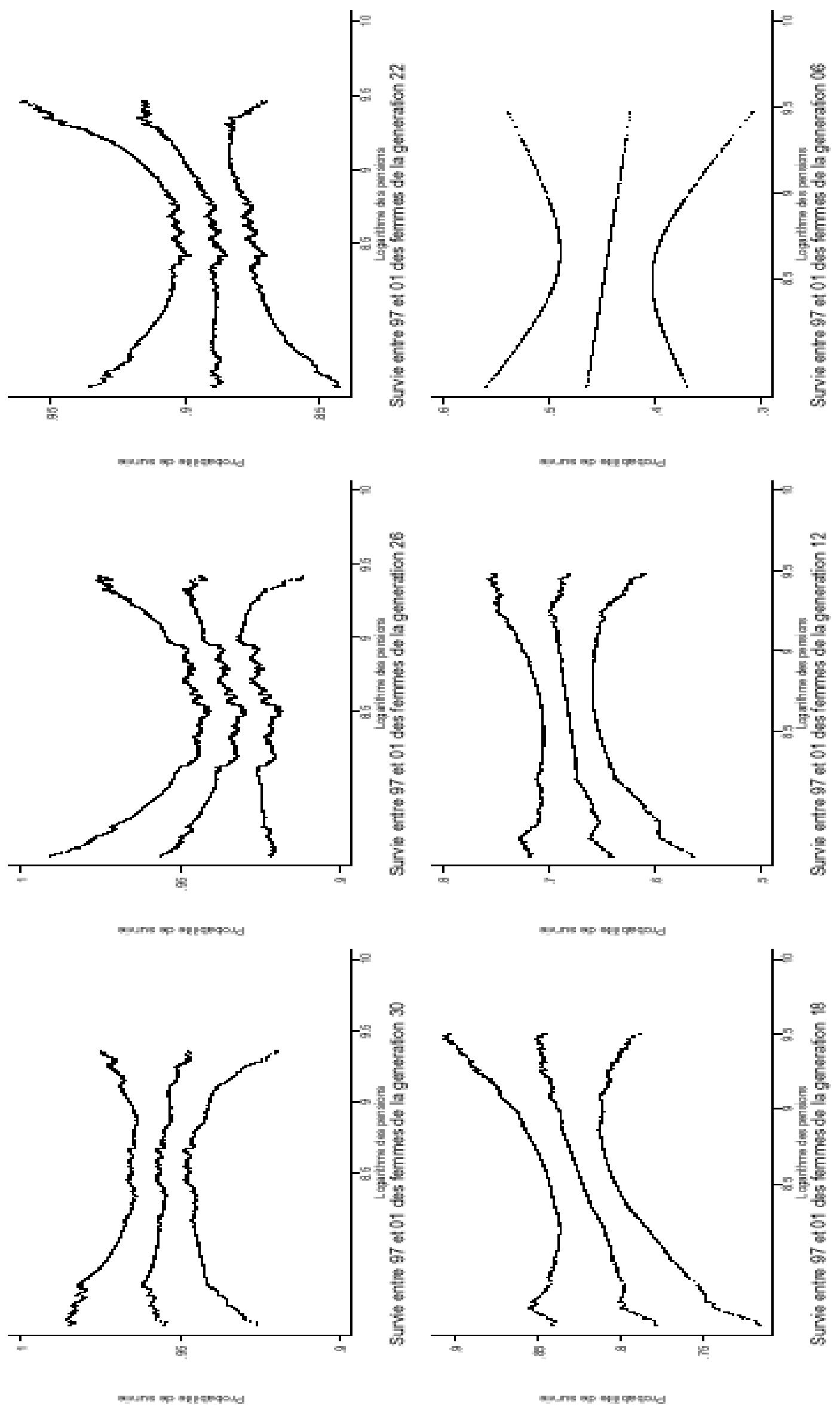